\title{
Kernos
}

Revue internationale et pluridisciplinaire de religion grecque antique

$16 \mid 2003$

Varia

\section{G. SFAMENI GASPARRO, Oracoli, Profeti, Sibille}

\section{Emilio Suárez de la Torre}

\section{(2) OpenEdition}

\section{Journals}

Édition électronique

URL : http://journals.openedition.org/kernos/848

DOI : $10.4000 /$ kernos. 848

ISSN : 2034-7871

Éditeur

Centre international d'étude de la religion grecque antique

Édition imprimée

Date de publication : 1 janvier 2003

Pagination : 376-377

ISSN : 0776-3824

Référence électronique

Emilio Suárez de la Torre, "G. sfameni gasparro, Oracoli, Profeti, Sibille», Kernos [En ligne], 16 | 2003, mis en ligne le 14 avril 2011, consulté le 22 septembre 2020. URL : http://journals.openedition.org/kernos/ 848 ; DOI : https://doi.org/10.4000/kernos.848 
Giulia Sfameni Gasparro, Oracoli, Profeti, Sibille. Rivelazione e salvezza nel mondo antico, Roma, Libreria Ateneo Salesiano, 2002. 1 vol. $16,5 \times 24 \mathrm{~cm}, 489$ p. (Biblioteca di Scienze Religiose, 171). ISBN : 88-213-0482-5.

Le titre de cet ouvrage ne rend que partiellement justice à la richesse thématique de son contenu. C'est plutôt le sous-titre qui nous permet d'envisager le parcour's auquel nous convie ce magnifique volume sur la religiosité de la période impériale, car les différents chapitres nous ouvrent largement des perspectives très enrichissantes sur le prophétisme, les croyances aux dieux guérisseurs, le concept de daimôn, les conceptions sur le destin et le culte d'Isis, sans oublier les nombreuses réflexions sur une multiplicité d'aspects de la religion grecque pendant les premiers siècles de l'empire romain qui foisonnent entre ces pages. Le fait que ce soit un recueil de travaux déjà publiés n'empêche pas d'apercevoir la remarquable cohérence de l'ensemble. À vrai dire le volume est un beau témoignage d'un parcours intellectuel rigoureux commencé une dizaine d'années avant sa publication, à l'occasion de la préparation d'un congrès, et qui est parfaitement expliqué par l'A. dans la "Prefazione » (p. 5-11). Précédé d'une "Nota introduttiva » (p. 15-21) de Biagio Amata, le contenu s'articule en huit chapitres, suivis d'un Appendice (en fait un compte rendu critique et une prise de position sur l'ouvrage de D.E. Aune, Prophecy in Early Christianity and the Ancient Mediterranean World, Grand Rapids, 1982, $1991^{2}$, dont l'insuffisante considération de la fonctionnalité religieuse et culturelle des phénomènes étudiés est mise en relief par l'A.).

Les chapitres I à IV développent surtout la thématique prophétique et oraculaire (c'est-à-dire la «révélation »), tandis que les suivants nous guident par le monde de la «salvezza»: le $\mathrm{V}^{\mathrm{e}}$ chapitre, sur Aelius Aristide, est une parfaite "cheville» entre la révélation et le salut, étant donné la foi de cet auteur en Asclépios et les moyens de communication avec le dieu qu'il nous décrit. Le chapitre I sert d'introduction générale à cette série ( Oracolo, divinazione, profetismo nel mondo greco-romano da Augusto alla fine del II secolo : un'introduzione al tema », p. 1-60). On commence par une intéressante problématique, le contraste (et les rapports) entre les consultations oraculaires 'publiques' et 'privées' (à propos du De Pythiae oraculis de Plutarque), qui ouvre des perspectives intéressantes d'approfondissement (I'A. soutient que les données obligent à corriger l'opinion de Plutarque). Ensuite l'A. nous guide à travers les noms et les textes les plus représentatifs du monde du prophétisme de cette période. Les données sur Apollonius de Tyane ou sur Alexandre d'Abonotique, la dévotion d'Aelius Aristide et les oracles " théologiques " sont présentés et analysés en fonction de la thématique à clévelopper.

Les chapitres suivants se partagent le traitement en détail des thèmes annoncés dans le premier chapitre. Le chapitre II ( La sibilla, voce del dio per pagani, ebrei e cristiani : un modulo profetico al crocevia delle fedi », p. 61-112) est une analyse approfondie de ce versant particulier du prophétisme ancien, en partant du contexte prophétique du monde gréco-romain et à travers l'étude du processus d'assimilation et de métamorphose de ce modèle «païen» par les juifs et les chrétiens jusqu'à saint Augustin. Le chapitre III («Plutarco e la religione delfica : il dio 'filosofo' e il suo esegeta », p. 113-148) est beaucoup plus qu'une étude des dialogues «pythiques» de Plutarque. On y trouve une importante méthode d'analyse de la pensée de Plutarque qui nous montre à quel point philosophie et théologie sont unies dans l'univers de ses croyances autour de la figure d'Apollon et de la «religion delphique ». J'oserais dire que, sans le prétendre (et sans l'exprimer à proprement parler), l'A. nous donne ici un bon instrument pour suivre le développement de la pensée néoplatonicienne, enrichie d'éléments variés et adaptée à l'expérience religieuse delphique de Plutarque. Le chapitre IV ( Alessandro di Abonotico, lo "pseudoprofeta' ovvero come costruirsi un'identità religiosa », p. 149-202) exploite de façon remarquable la valeur historique du témoignage que nous donne Lucien sur ce personnage extraordinaire, qui devient une impressionnante synthèse de la religiosité de son époque. Le grand mérite de cette étude est précisément la richesse des rapports qu'elle établit entre le personnage et ses activités, d'un côté, et la réalité religieuse contemporaine, de l'autre, en appuyant le cadre qui se dégage des sources littéraires avec une formidable batterie de données provenant des sources secondaires et de l'épigraphie. Dans le chapitre V (« Elio Aristide e Asclepio, un retore e il suo dio : salute del corpo e direzione spirituale », p. 203-253), on trouvera une révision des Discours sacrés qui place avec 
précision le profil de ce rhéteur mégalomane et hypocondriaque dans le cadre contemporain du culte d'Asclépios et des doctrines sur l'interprétation des songes; l'A. souligne la conviction d'Aristide d'être un intermédiaire entre son dieu et les hommes, ainsi que la tendance hénothéiste que reflète le rapprochement Zeus-Asclépios qui se dégage des ses discours.

Le Destin et la Fortune sont le sujet d'étude du chapitre VI ( Daimon et Tyche nell'esperienza religiosa dell'uomo ellenistico : strutture ideologiche e pratiche cultuali », p. 255-301). Le concept de daimon, en singulier et en pluriel, est étudié par l'A. depuis Homère d'une façon remarquablement précise. Cette partie sert d'introduction à l'examen de la documentation concernant l'Agatbos Daimon, la Tyche et l'Agatbe Tyche, dans un effort pour nuancer et délimiter les traits de chaque culte, ainsi que de fixer le procédé d'entrecroisement de ces conceptions (avec une rigoureuse présentation des données épigraphiques par régions). Les deux derniers chapitres abordent deux aspects différents, mais complémentaires, du culte isiaque. D'un côté le rapprochement d'Isis et de la déesse Fortune (ch. VII, «Iside-Fortuna : Fatalismo e divinità sovrane del destino del mondo ellenistico-romano ", p. 303-325), ce qui mène l'A. non seulement à suivie les traces de la conjonction Isis-Fortuna (et en tant que fortuna videns), mais aussi de sa qualité de divinité cosmique. Les conclusions tirées des sources majoritairement littéraires (tout spécialement Apulée) sont confrontés à d'autres témoignages qui confirment l'accord avec la religiosité populaire. D'un autre côté, ce sont les aspects guérisseurs et de salut de la déesse qui composent le sujet d'étude de l'A. (ch. VIII, Iside Salutaris : aspetti medicali e oracolari del culto isiaco tra radici egiziane e metamorfosi ellenica ", p.327-342), qui souligne l'accord entre la tradition égyptienne sur les pouvoirs médicaux d'Isis et les pratiques magiques avec les courants religieux grecs de cette période.

Le résumé du contenu que je viens de faire ne donne qu'une idée très faible des vertus de cet ouvrage. Douée d'une remarquable formation historico-religieuse, avec une maîtrise incontestable dans la recherche des sources les plus variées, l'A. nous livre un très beau recueil. Ce n'est point une simple addition d'articles plus ou moins proches dans leur thématique, mais un ensemble bien atticulé qui forme une monographie indispensable pour comprendre la religiosité des premiers siècles de notre ère en général et pour la thématique oraculaire et du salut en particulier.

Emilio Suárez de la Torre (Universidad de Valladolid)

Françoise LÉToublon (coordonné par), Le mythe d'Orphée dans les métamorphoses d'Ovide, Paris, Adapt Éditions, 2001. 1 vol. $15 \times 21 \mathrm{~cm}, 128 \mathrm{p}$.

La première moitié de l'ouvrage, intitulće Orphée et le mythe de la poésie, est l'œuvre de la coordinatrice, Françoise Létoublon. Elle est divisée en quatre parties : 1 . Le personnage d'Orphée: un mythe sans substance? 2. Orphée avant Ovide. 3. Orphée dans la composition des Métamorphoses. 4. Les thèmes centraux du mythe d'Orphée. Elle s'achève par une bibliographie de 32 ouvrages : les articles ont été écartés car les A. cherchent moins à faire œuvre d'érudition qu'à ouvrir des pistes de recherche et s'adressent à un public plus étendu que celui des antiquisants (mais ceux-ci trouveront aussi de quoi nourrir leur réflexion dans ce petit ouvrage). L'A. note que le mythe d'Orphée est un mythe grec sans textes grecs des époques archäque et classique en dehors de fragments et d'allusions. On ne peut que regretter la disparition de la tragédie d'Eschyle intitulée les Bassarai, dont il reste vingt-neuf mots en quatre fragments et qui, semble-t-il, racontait ou représentait la mort d'Orphée. Les textes complets sont tous postérieurs à 200 ap. J.-C. Il s'agit d'une collection d'Hymnes, d'une épopée intitulée Argonautiques orphiques et d'un recueil poétique sur la puissance magique des pierres appelé Litbika. En revanche, les représentations figurées et les inscriptions sont abondantes et commencent dès le $\mathrm{vl}^{\mathrm{e}}$ siècle av. J.-C. De plus des découvertes archéologiques en provenance de tombes viennent enrichir le corpus: papyrus de Derveni sur des mystères orphiques, lamelles d'or ou plaques en os donnant des instructions pour le voyage dans l'au-delà. Le problème qui se pose aux chercheurs est qu'il est souvent difficile de faire le départ entre les doctrines dionysiaques, pythagoriciennes ou orphiques, tantôt proches, tantôt antagonistes. 